\title{
Telemedicine Digital Phonocardiography: Cost-Effective Strategies in Heart Failure Screening and Monitoring
}

\author{
S Khoor ${ }^{1}$, I Kovacs ${ }^{1}$, K Fugedi ${ }^{1}$, G Horvath ${ }^{1}$, \\ E Domijan $^{2}, \mathrm{M}$ Domijan $^{2}$ \\ ${ }^{1}$ Szent Istvan Hospital, Budapest, Hungary \\ ${ }^{2}$ UVA Corporation, Toronto, Canada
}

\begin{abstract}
Three studies were performed assessing the clinical value of digital electro- and phonocardiography (dECG, $d P C G$ ) with telemedicine application. In the first study, some Doppler echocardiographic parameters (ejection fraction, aortic Vmax, the grade of mitral and tricuspid regurgitation) were estimated from the spectral amplitude value of 170 time-frequency cells of the TriTest dPCG using multivariate discriminant analysis of 584 cardiac patients (292 for the training, and 292 for the test set). A cost analysis of heart failure (HF) screening in various populations was performed on 452 subjects. The greatest cost-savings (Euro / one HF patient) was found in the combined use of $d E C G$ and $A P C G$ compared with the TE screening alone (mean: 82.4 CI-95\%: 69.5-96.4 versus mean: 230.1 CI-95\%: 196.5-254.4; $p<0.001)$. In the third study, during the 24 months telemonitoring of serious heart failure patients, 124 hospital days charge was saved, comparing the two, 29-29 patients' groups.
\end{abstract}

\section{Introduction}

In the last two decades, Tavel has written almost everything about the clinical use of auscultation and phonocardiography $[1,2]$. Our works started from this point, introducing more sophisticated computer application, and expanding the patients data set with digital phonocardiograms (dPCG), electrocardiograms (dECG), and the traditional transesophageal echocardiograms (TTE) $[3,4]$. From the first work on spectral analysis of the PCG [5] to the last [6], many efforts were made [7-10], mainly in pediatrics [11-13], without any significant change in everyday clinical use. The aim of our studies was to expand the clinical use of dPCG with advanced computer technique (signal pre-, and post-processing, 3D visualization of the heart sounds and murmurs, communication) combined with telemedicine application. By the cardiology point of view, the complex management of heart failure (HF) patients is essential, but time and cost consuming. The cost of the hospitalization is the dominant component, thus every cost-efficacy/efficiency analysis is based on it. The wide range of telemedicine applications in cardiology could be found in the literature, lesser in the area of signal processing (almost all in computerized electrocardiography), and a few of using phonocardiograms [14-16]. The telemonitoring of (mostly advanced) heart failure patients is a very promising way in the complex and cost-saving care [17-19]. Screening the left ventricular dysfunction would be very important, because the heart failure is associated with high morbidity, mortality, and cost [20-22]. In this paper the cost analysis of heart failure management (screening and monitoring) with or without telemedicine application is performed.

\section{Methods}

In the first study 584 cardiac patients (292 for the training and 292 for the test set) were enrolled. The Doppler echocardiographic parameters (ejection fraction, aortic Vmax, the grade of mitral and tricuspid regurgitation (I-III)) were estimated from the spectral amplitude values of 170 time-frequency cells of the dPCG.

In the second study, the patients were recruited from the general population of a small town in Hungary (7400 inhabitants. The study population consists of a general population (GPg; N=192), a low-risk group (LRg; $\mathrm{N}=113$ ), and a high-risk group (HRg; $\mathrm{N}=147)$. High risk patients: any of IHD, hypertension (blood pressure> 160/100), diabetes, peripheral/cerebral vascular disease, and heavy alcohol use (alcoholism or 40 units alcohol per week). Low risk patients are defined as patients without target organ damage, but are on higher cardiovascular risk using the European Risk Score (value $>5.0$ ). Four screening strategies were compared by sensitivity and cost-effectiveness analysis: Strategy 1 (Str_1): all subjects to undergo TE (gold-standard strategy); Strategy 2 (Str_2): all subjects with an abnormal ECG were undergone TE; Strategy 3 (Str_3): all subjects with an 
abnormal dPCG were undergone TE; Strategy 4 (Str_4): all subjects with an abnormal ECG and abnormal dPCG were undergone TE. In the statistical analysis the screening characteristics and cost-effectiveness were calculated in the three groups. The screening characteristics and cost-effectiveness defined as the cost per case of sHF and of dHF found. Strategy 1, or TE alone, was the gold-standard strategy. To account for multiple testing, $\mathrm{P}<0.01$ was taken as significant.

58 patients were enrolled in the third study, where the cost-effective strategies of telemedicine monitoring were analyzed. All of these patients had serious (NYHA IIIIV) heart failure (HF). Twenty-nine patients were monitored in every 4 weeks by the telemedicine method (12-lead ECG, 30-minute ECG monitoring, dPCG; either in the GP's office, or at home), and 29 age-matched patients consist the control group.

The echocardiographic parameters were measured by the standard mode; heart failure was defined according to European guidelines. The following dPCG parameters were determined by windowed FFT analysis of dPCG (TriTest device, sampling rate $1 \mathrm{kHz}$, in the range of 20$12000 \mathrm{kHz}$ ): third, fourth heart sound, systolic murmur at the apex. The dPCG recordings were stepwise processed: general bandpass, adaptive by the segments of cardiac cycle, and median filtering were used.

Data were derived from the nationwide administrative dataset of the National Health Insurance Fund Administration (OEP); the cost of is calculated from the annual resource use (DRG cost-weights, heart failure cases and hospital days).

\section{Results}

The echocardiographic aortic Vmax (detecting systolic failure), the ejection fraction (EF), the rate of mitral and tricuspid regurgitation (MR, TR) were estimated using SPSS (V15.0) multivariate discriminant analysis (MDA) module. These output variables converted into three discrete values, and the process was the following (example of the estimation of the three grades of MR). The model (MDA, Wilks' method) chooses the best input parameters from the 170 time-frequency amplitude values from one cardiac cycle of dPCG. The Wilks's statistic selects the best parameters (found seven) and the unstandardized canonical discriminant function coefficients were determined. The linear multivariate discriminant score equation is calculated from the measured parameters, and these coefficients, either the training, or the test sets. The model chooses 17 input parameters for the Vmax, 9 for the EF, and 6 for the TR. The statistics of classification: for Vmax: Wilks's lambda: 0.111 , chi-square: 274.6 , degree of freedom: 34 ; for EF: $0.349,154.1,15$; for MR: 0.416, 113.0, 14; for
TR: $0.535,81.6,12$, respectively; all $\mathrm{p}<0.0001$.

Table 1. shows the classification results of MDA for Vmax, EF, MR, and TR.

Table 1. Classification results of the four TTE parameters. Orig $\%=$ original groups, Pred $\%=$ predicted groups.

\begin{tabular}{|l|rrrr|}
\hline & \multicolumn{4}{|c|}{ Pred \% } \\
\hline Orig\% & Group & \multicolumn{1}{|c}{ \% } & $\mathbf{2}$ & $\mathbf{3}$ \\
\hline Vmax & $\mathbf{1}$ & 97.5 & 2.5 & 0 \\
\hline & $\mathbf{2}$ & 30.8 & 69.2 & 0 \\
\hline & $\mathbf{3}$ & 0 & 0 & 100 \\
\hline EF & $\mathbf{1}$ & 78.4 & 10.4 & 11.2 \\
\hline & $\mathbf{2}$ & 29.3 & 67.9 & 4.8 \\
\hline & $\mathbf{3}$ & 6.6 & 23.9 & 69.5 \\
\hline MR & $\mathbf{1}$ & 85.0 & 12.4 & 2.7 \\
\hline & $\mathbf{2}$ & 33.3 & 66.7 & 0 \\
\hline & $\mathbf{3}$ & 14.3 & 14.3 & 71.4 \\
\hline TR & $\mathbf{1}$ & 75.0 & 21.6 & 3.4 \\
\hline & $\mathbf{2}$ & 38.5 & 61.5 & 0 \\
\hline & $\mathbf{3}$ & 14.3 & 0 & 85.7 \\
\hline
\end{tabular}

The prevalence of sHF was $0.34 \%$ in the LRg vs. $9.9 \%$ in the HRg $(\mathrm{P}<0.001)$, and of dHF was $1.1 \%$ and $12.4 \%$, respectively $(\mathrm{P}<0.001)$. The screening characteristics of combined dECG and dPCG (sensitivity, specificity, positive and negative predictive value were: in $\mathrm{HRg}$ with sHF: 93.0, 68.8, 91.5, 73.3; with dHF: 82.4, 45.2, 67.3, 65.1; LRg with sHF: 74.9, 55.0, 89.7, 42.3; with dHF: 74.7, 29.4, 65.1, 29.6; in GPg with sHF: 78.5, 41.7, 64.6, 58.8; with dHF: $67.5,38.4,60.4,52.2$, respectively.

The unit costs were estimated at $175 €$ for TE, 34 for dPCG, and 15 for the dECG. The costs per case of systolic and/or diastolic HF found using the most likely current estimate of test costs. The cost per case of sHF found of Str 1. Was 8387.5 $€$ of low-risk patients and $230.1 €$ of high-risk patients. The cost per case value of dHF in Str 1. were $7994.3 €$ in the LRg and $221.9 €$ in the HRg. Comparing the four strategies, the costs were (expressed in percentage of Str 1.) for Str 2.: 23.6\%, Str 3.: $19.1 \%$, Str 4.: $42.5 \%$, Str 5.: $29.0 \%$. The same values for dHF were in the LRg 19.4, 16.8, 38.6, 14.4; in the HRg were 21.4, 14.2, 36.7, and 16.8, respectively.

The telemedicine screening of the patients by ECG and APCG could produce great cost-savings compared with the TE screening alone (Str4: mean: 82.4 CI-95\%: 69.5-96.4 versus Str1: mean: 230.1 CI-95\%: 196.5-254.4; $\mathrm{p}<0.001)$.

In the third study, there were not any significant differences between the two groups (G2: Usual Care, G1: Telemedicine): in Group G2: age $64.8 \pm 8.5$, female: 13 (44.8\%), sHF: $21(72.4 \%)$, dHF: $8(27.6 \%)$, prior myocardial infarction: 19(65.5), NYHA II: 8(27.6), 
NYHA III: 18(62.1), NYHA IV: 3 (10.3\%), therapy with beta blocker $17(58.6 \%)$, with ACEI 24(82.7\%), with ARB 5(17.2\%); in Group G1: age $63.3 \pm 8.1$, female: 14 (48.3\%), sHF: $21(72.4 \%)$, dHF: $8(27.6 \%)$, prior myocardial infarction: 17(58.6), NYHA II: 7(24.1), NYHA III: 18(62.1), NYHA IV: 4 (13.8\%), therapy with beta blocker 18(62.1\%), with ACEI 25(86.2\%), with ARB 5(17.2\%). The primary outcome measurements of the 2-years follow-up of the telemedicine study are showed in Table 2.

Table 2. Primary outcomes of the 2 years follow-up. hosp $=$ hospitalization, days/admission $=$ hospital duration per one admission, percent of days in hospital: days in hospital/potential days.

\begin{tabular}{|lcc|}
\hline Variable & Usual Care & Telemedicine \\
\hline Patients & 29 & 29 \\
\hline Patients hosp. & $17(58.6 \%)$ & $12(41.4 \%)$ \\
\hline Number of hosp. & 78 & 55 \\
\hline Days in hospital & $968(4.5 \%)$ & $546(2.3 \%)$ \\
\hline Days/admission & 12.4 & 9.9 \\
\hline Heart failure hosp. & $46(58.9 \%)$ & $31(56.3 \%)$ \\
\hline Other CV hosp. & $21(26.9 \%)$ & $15(27.4 \%)$ \\
\hline Non CV hosp. & $11(14.2 \%)$ & $9(16.3 \%)$ \\
\hline HF hosp. days & $570(2.7 \%)$ & $307(1.4 \%)$ \\
\hline HF hosp. duration & 11.8 & 8.2 \\
\hline Deaths & $10(34.4 \%)$ & $6(20.6 \%)$ \\
\hline Circulatory failure & 7 & 4 \\
\hline Sudden death & 3 & 1 \\
\hline Other death & 2 & 1 \\
\hline
\end{tabular}

At 2 years, $79.4 \%$ of patients ( 23 of 29 patients) in telemedicine group (G1) were alive compared with $65.6 \%$ of those in usual care group (G2) (19 of 29 patients). A Kaplan-Meier graph indicated, that the curves began to widen after 10 months and remained divergent at 2 years (Figure 1).

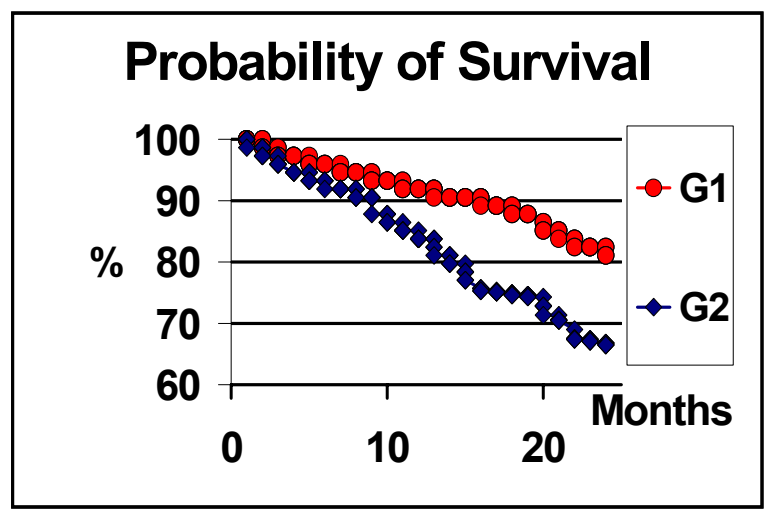

Figure 1. The probability of survival. G1= telemedicine group, $\mathrm{G} 2=$ usual care group.
The Hungarian DGR cost-weight of one (1.0) costweight is 132,600 HUF (526.2 €). The DRG cost-weight of heart failure is 0.915 ; the normative days of $\mathrm{HF}$ are 9.0. Table 3. shows the data of the 2-years telemedicine follow-up cost-analysis of the 58 heart failure patients.

Table 3. Telemedicine cost-analysis. $\mathrm{No}=$ number, $\mathrm{G} 1=$ telemedicine group, $\mathrm{G} 2=$ usual care group, $\mathrm{HC}=$ hospital cost, $\mathrm{C}=$ cost, $\mathrm{Nu}=$ Nurse, $\mathrm{TM}=$ telemedicine, $\mathrm{GP}=$ general practitioner, $\mathrm{Ca}=$ cardiologist, $\mathrm{THC}=2$ years total hospital cost, $\mathrm{HF}=$ heart failure, $\mathrm{NNV}=$ number of nurse visit, NTMV = number of telemedicine visit, $\mathrm{NGPV}=$ number of general practitioner visit, $\mathrm{NCV}=$ number of cardiologist visit, NTMV $=$ number of cardiologist visit, NTMC $=$ number of telemedicinedriven extra cardiologist visit, $\mathrm{TC}=2$ years total cost.

\begin{tabular}{|lcrr|}
\hline Parameter & No. & Cost HUF & Cost $€$ \\
\hline 1 day HC & & 13481 & 53.5 \\
\hline Nu visit C & & 2200 & 8.7 \\
\hline TM visit C & & 2700 & 10.7 \\
\hline GP visit C & & 3600 & 14.3 \\
\hline Ca visit C & & 7100 & 28.2 \\
\hline THC of G1 & & 340404.5 & 29128.6 \\
\hline THC of G2 & & 13038823.2 & 51741.4 \\
\hline G1:HF THC & & 4532312.2 & 17985.4 \\
\hline G2:HF THC & & 9544548 & 37875.2 \\
\hline NNV in G1 & 743 & 1634600 & 6486.5 \\
\hline NNV in G2 & 768 & 1689600 & 6704.8 \\
\hline G1:NTMV & 704 & 1900800 & 7542.9 \\
\hline NGPV in G1 & 468 & 1684800 & 6685.7 \\
\hline NGPV in G2 & 511 & 1839600 & 7300.0 \\
\hline NCV in G1 & 178 & 1263800 & 5015.1 \\
\hline NCV in G2 & 143 & 1015300 & 4029.0 \\
\hline G1:NTMC & 296 & 2101600 & 8339.7 \\
\hline TC of G1 & & 15926004.5 & 63198.4 \\
\hline TC of G2 & & 17583323.2 & 69775.1 \\
\hline
\end{tabular}

Comparing the data of the last two rows, the costsavings of 122.9 hospital days were found.

\section{Discussion and conclusions}

The first study showed that the method of multiple discriminant analysis could adequately model the timefrequency measurements of $\mathrm{dPCG}$ and echocardiographic data in a population of various severity of cardiac disease. The second study has shown that screening high-risk subjects is always more cost-effective than screening general population subjects and much more cost-effective than screening low-risk subjects. Using the ECG and digital phonocardiography methods to predict heart failure in various (general population subjects, high-, and low-risk patients) groups, significant cost-savings would 
be achieved comparing echocardiography alone. The third study suggests that telemedicine heart failure service with digital electro- and phonocardiography, can reduce mortality substantially in patients with heart failure. The reduction in mortality is achieved with a decrease in the duration of time spent in hospital. This method a cost-effective solution for the delivery of expert care for patients with heart failure and saves approximately 120 hospital day's charge during two years follow-up of 58 patients. The three studies showed two special methods of internet use in telemedicine application: firstly, how to register $\mathrm{dECG}$ and $\mathrm{dPCG}$ data far from the cardiologist, secondly how to execute the complex math calculations using the store-and-forward method via the internet. The digital phonocardiography would be a useful method in population screening and monitoring left ventricular dysfunction. This kind of telemedicine application significantly raises the accessibility of general patient population to the adequate management of various heart diseases.

\section{References}

[1] Tavel ME. Cardiac auscultation: a glorious past - but does it have a future? Circulation 1996;93:1250-53.

[2] Tavel ME. Cardiac auscultation: a glorious past-and it does have a future! Circulation 2006;113:1255-59.

[3] Kail E, Khoor S, Kail B, Fugedi K. Internet digital phonocardiography in clinical settings and in population screening. Computers in Cardiology 2004;31:501-4.

[4] Kail E, Khoor S, Fugedi K, Kovacs I, et al. Expert system for phonocardiographic monitoring of heart failure patients based on wavelet analysis. Computers in Cardiology 2005;32:833-6.

[5] McKusick VA, Webb GN, Humphries JA, Reid JA. On cardiovascular sound: further observations by means of spectral phonocardiography. Circulation 1958;11:849-70.

[6] Seyal MS. The Virtual Cardiac Patient: A multimedia guide to heart sounds and murmurs. JAMA 2007; 297(2):217-18.

[7] Rangayyan RM, Lehner RJ. Phonocardiogram signal analysis: a review. Crit Rev Biomed Eng 1988;15:211-236.

[8] Durand LG, Pibarot P. Digital signal processing of the phonocardiogram: review of the most recent advancements. Crit Rev Biomed Eng 1995;23(3):163-219.

[9] Xuan Z, Durand LG, Senhadji L, et al. H.C. Analysis synthesis of the phonocardiogram based on the matching pursuit method . IEEE Trans Biomed Eng 1998;45:962-71.

[10] Debbal SM, F. Bereksi-Reguig F. Graphic representation and analysis of the PCG signal using the continuous wavelet transform. The Internet Journal of Bioengineering. 2007;2:2.

[11] Barschdorff D, Femmer U, Trowitzsch E. Automatic phonocardiogram signal analysis in infants based on wavelet transforms and artificial neural networks. Computers in Cardiology 1995;7:753-756.

[12] Balster DA, Chan DP, Rowland DG, AllenHD. Digital acoustic analysis of precordial innocent versus ventricular septal defect murmurs in children. Am J Cardiol 1997;79:1552-55.

[13] Bhatikar SR, DeGroff C, Mahajan RL. A classifier based on the artificial neural network approach for cardiologic auscultation in pediatrics. Artif Intell Med 2005;33(3):25161.

[14] Bhatikar SR , Mahajan RL, DeGroff C. A novel paradigm for telemedicine using the personal bio-monitor. Biomed Sci Instrum. 2002;38:59-70.

[15] Brusco M, Nazeran H. Digital phonocardiography: a PDAbased approach. Conf Proc IEEE Eng Med Biol Soc 2004 ;3:2299-302.

[16] Kovacs F, Horvath C, Torok M, Hosszu G. Long-term Phonocardiographic Fetal Home Monitoring for Telemedicine Systems. Proc IEEE Eng Med Biol Soc. 2005;4(1):3946-49.

[17] Louis AA, Turner T, Gretton M, Baksh A, Cleland JGF. A systematic review of telemonitoring for the management of heart failure. Eur J Heart Fail 2003;5:583-90.

[18] Goldberg LR, Piette JD, Wals MN, et al. Randomized trial of a daily electronic home monitoring system in patients with advanced heart failure: the Weight Monitoring in Heart Failure (WHARF) trial. Am Heart J 2003;146:70512.

[19] Cleland JGF, Louis AA, Rigby AS, at al. Noninvasive home telemonitoring for patients with heart failure at high risk of recurrent admission and death: The Trans-European Network-Home-Care Management System (TEN-HMS) study. JACC 2005;45;1654-1664.

[20] McMurray JV, McDonagh TA, Davie AP, et al. should we screen for asymptomatic left ventricular dysfunction to prevent heart failure? Eur Heart J 1998;19:842-6.

[21] Khunti K, Squire I, Abrams KR, Sutton AJ. Accuracy of a 12-lead electrocardiogram in screening patients with suspected heart failure for open access echocardiography: a systematic review and meta-analysis. Eur J Heart Fail 2004;6:571-6.

[22] Galasko GIW, Barnes, SC, Collinson P, et al. What is the most cost-effective strategy to screen for left ventricular systolic dysfunction: natriuretic peptides, the electrocardiogram, hand-held echocardiography, traditional echocardiography, or their combination? Eur Heart J 2006;27:193-200.

Address for correspondence

Sandor Khoor MD PhD

Szent Istvan Hospital

$3^{\text {rd }}$ Dept Int Med

Nagyvarad ter 1

Budapest, Hungary, H-1096

skhoor@gmail.com

sandor.khoor@uva.ca 